\title{
A influência do método estratégico jejum intermitente para o emagrecimento saudável: uma revisão
}

\author{
An influence of the strategic method intermittent fasting \\ for healthy weight loss: a review
}

Marília Celeste Brito da Cunha', Edson Douglas Silva Pontes', Mayara Gabrielly Germano de Araújo', Jaielison Yandro Pereira da Silva', Nilcimelly Rodrigues Donato 2

'Universidade Federal de Campina Grande

¿Universidade Federal de Campina Grande. Centro de Educação e Saúde. Unidade Acadêmica de Saúde. Curso de Graduação em Nutrição

Email para contato: Nilcimelly Rodrigues Donato - mellydonato@gmail.com

\section{Resumo}

Baseado na elevada incidência de excesso de peso e obesidade em todo o mundo, dietas para emagrecimento são fortemente requisitadas pela população, a exemplo da dieta do jejum intermitente, que ganhou destaque ficando conhecida como uma "dieta da moda". Dessa forma, objetivou-se realizar uma revisão na literatura sobre o jejum intermitente analisando seus possíveis benefícios e efeitos adversos. Para isso, foi conduzida uma busca bibliográfica nas bases de dados: PubMed, Google Acadêmico, MEDLINE e Periódicos CAPES. Foram incluídos nessa pesquisa apenas artigos que envolvessem seres humanos publicados em língua inglesa e portuguesa no período de 2007 a 2017. Ao final, foram encontrados 12 artigos de ensaios clínicos e/ou randomizados em humanos considerando 4 subclasses do método jejum intermitente. Todos os estudos analisados descrevem alterações nos parâmetros de composição corporal após intervenção em curto prazo. Novos estudos envolvendo seres humanos são necessários para avaliar eficácia, viabilidade e riscos à longo prazo.

Palavras-chave: Perda de peso. Obesidade. Composição corporal.

\begin{abstract}
Based on the high incidence of overweight and obesity worldwide, diets for weight loss are strongly requested by the population, such as the intermittent fasting diet, which gained prominence and became known as a "fad diet". Thus, the objective was to conduct a review of the literature on intermittent fasting, analyzing its possible benefits and adverse effects. For this, a bibliographic search was conducted in the databases: PubMed, Google Scholar, MEDLINE and Periódicos CAPES. Only articles involving human beings published in English and Portuguese between 2007 and 2017 were included in this research. At the end, 12 articles from clinical and / or randomized trials in humans were found considering 4 subclasses of the intermittent fasting method. All analyzed studies describe changes in body composition parameters after short-term intervention. Further studies involving humans are needed to assess long-term effectiveness, viability and risks.
\end{abstract}

Keywords: Weight loss. Obesity. Body composition. 


\section{INTRODUÇÃO}

Todos os animais necessitam de alimento para sua sobrevivência. Contudo, nota-se que desde a era paleolítica períodos escassos de alimentos foram vivenciados, fato que impulsionou um processo evolutivo do sistema fisiológico e comportamental dos organismos, possibilitando a supervivência durante esses episódios de ausência ou carência alimentar ${ }^{1,2}$.

De acordo com a literatura, sobreviver em tempos de quase e/ou nenhuma ingestão de alimentos é possível devido à presença de mecanismos e funções realizadas por órgãos fundamentais no processo adaptativo, a exemplo do fígado ${ }^{3}$. Esse órgão é capaz de estimular a glicogenólise e a gliconeogênese para manutenção da glicemia. Quando os níveis de glicose estão quase que inexistentes, o fígado é capaz de fornecer energia através da mobilização das gorduras hepáticas e produção de corpos cetônicos4.

Apesar de ser um processo já adaptado e vivenciado pela história humana, "atualmente o estado de jejum passou a ser, em geral, um processo opcional, uma vez que há raramente privação alimentar ou escassez de alimentos. Neste sentido, surgiram diferentes estratégias nutricionais que se baseiam no estilo de vida dos nossos antepassados, uma dessas estratégias é o jejum intermitente"1.

Em geral, o jejum intermitente (JI) é definido como um processo de abstinência de alimentos por períodos de duração variável, podendo ser aplicado como um hábito alimentar no contexto de um padrão dietético de promoção a saúde ou como também para fins religiosos, a exemplo do Ramadã ${ }^{3,4}$.

Por ser uma estratégia intervencionista, o JI atraiu uma atenção devido a estudos experimentais em que indivíduos são submetidos a diferentes períodos de jejum e assim podem obter correções de anormalidades metabólicas, tais como: obesidade, diabetes, doenças cardiovasculares ${ }^{5}$ câncer e doenças neurodegenerativas ${ }^{6,7,8}$.

Estudos em humanos tem relacionado o JI com a perda de peso corporal estimada em uma faixa de aproximadamente $4 \%$ a $10 \%$ em períodos de curta e longa duração $0^{9,5,10,11}$.

Com base nesses dados, o JI pode ser um método com potenciais mecanismos para redução do peso corporal, visto a importância de novas estratégias eficazes para tratar problemas de saúde relacionados ao excesso de peso e obesidade ${ }^{12}$. 
Marília Celeste Brito da Cunha, Edson Douglas Silva Pontes, Mayara Gabrielly Germano de Araújo, Jaielison Yandro Pereira da Silva, Nilcimelly Rodrigues Donato | A influência do método estratégico jejum intermitente para o emagrecimento saudável: uma revisão

Portanto, o objetivo desse trabalho é fornecer uma revisão integrativa de pesquisas realizadas em humanos, que explicam como o JI pode beneficiar o processo de emagrecimento e quais limites e cuidados devem ser tomados para a sua utilidade.

\section{MÉTODO}

Trata-se de uma revisão integrativa desenvolvida em cinco fases: 1 . Definição da pergunta norteadora; 2. Busca da evidência; 3. Revisão e seleção dos estudos; 4. Avaliação da qualidade dos estudos e 5. Apresentação dos resultados ${ }^{13}$.

Após definir a pergunta norteadora ( $O$ jejum intermitente pode ser eficaz para procedimento de perda de peso corporal saudável?), realizou-se a pesquisa por meio de base de dados: PubMed, Medline, Google Acadêmico e Periódico CAPES. Foram incluídos artigos científicos em língua inglesa e portuguesa revisados por pares, com foco nos que investigam a utilização do jejum intermitente e suas influências no processo de emagrecimento corporal, publicados entre os anos de 2007 a 2017. Para busca, foram utilizadas as palavras chaves: 1) weight loss; 2) fasting 5:2; 3) Ramadã; 4) Alternate fasting day; 5) power restriction de forma isolada e conjugada, em língua inglesa.

Todos os trabalhos foram avaliados de acordo com a qualificação dos periódicos, por meio da ferramenta Qualis-Periódicos disponível pela plataforma Sucupira entre Classificações de Periódicos Quadriênios, 2013 a 2016, com áreas de avaliação variando entre Nutrição, Medicina I, II , III, Ciências Biológicas I, II, III, Educação Física e Saúde Coletiva. Foram excluídas pesquisas experimentais em animais e os trabalhos publicados fora da delimitação temporal estabelecida.

Para a apresentação dos resultados, gráficos foram elaborados com auxílio do Microsoft Word Excel 2013. As demais partes dos dados como efeitos adversos foram apresentados de forma discursiva.

\section{RESULTADOS}

Após a sistematização dos dados, foram encontrados ao todo, 12 trabalhos que estudaram humanos, sendo 5 trabalhos de Jejum de dia alternado (JDA) com restrição parcial de energia (abarcando um total de 136 pessoas) e 1 com restrição completa (18 indivíduos). Dois trabalhos sobre jejum modificado 5:2(JM) envolvendo, ao todo, 170 participantes. Dois estudos sobre o jejum intermitente 
Marília Celeste Brito da Cunha, Edson Douglas Silva Pontes, Mayara Gabrielly Germano de Araújo, Jaielison Yandro Pereira da Silva, Nilcimelly Rodrigues Donato | A influência do método estratégico jejum intermitente para o emagrecimento saudável: uma revisão

religioso (JIR), tratando-se de jejum do Ramadã (250 indivíduos), e duas pesquisas sobre jejum por restrição de tempo (JRT) (27 pessoas).

Após a sistematização da qualidade dos periódicos dos trabalhos utilizados nessa pesquisa, verificou-se que os trabalhos corresponderam às áreas de Nutrição; Medicina I, II, III; Ciências Biológicas I, II, III; Educação Física e Saúde Coletiva. Observou-se que a maioria dos trabalhos recebeu classificação "A1" e que um trabalho foi classificado com " $C$ ". Tendo em vista esse resultado, o presente estudo foi embasado quase em sua totalidade por pesquisas de fundos consideráveis, fato que se faz importante para assegurar a confiança pelos resultados que foram expostos.

Avaliando a subclasse JDA, um estudo realizado com mulheres obesas ou com sobrepeso, foi observado que um jejum de dia alternado por 6 semanas foi suficiente para induzir a perda de peso de $84,3 \pm 11,44 \mathrm{~kg}$ para $78,3 \pm 10,18 \mathrm{~kg}(6 \mathrm{~kg})$, reduzindo significativamente o Índice de Massa Corporal (IMC) e a massa gorda dos participantes, bem como tendência em diminuir os níveis médios de lipídios sanguíneos em jejum, embora que não significativamente 5 . Os valores reduzidos de lipídeos (LDL, Colesterol total e triglicerídeos) achados nesse estudo, podem ser comparados a outro trabalho no qual também houve uma redução desses parâmetros, destacando apenas uma diferença entres os valores de HDL, que no primeiro trabalho citado apresentou aumento e na segunda pesquisa os valores de HDL diminuíram ${ }^{14}$.

Em outro estudo de JDA de 10 semanas, utilizando protocolo de dieta com quantidade mais alta de lipídios ( $45 \%$ ) e dieta com uma quantidade mais baixa de gordura (25\%) em relação ao outro grupo, observou-se uma diminuição de IMC, perda de peso corporal, sendo 4,3 $\pm 1,0 \mathrm{~kg}$ o resultado dos que consumiram dieta com conteúdo maior de gordura e 3,7 $\pm 0,7 \mathrm{~kg}$ o resultado do grupo que consumiu dieta com menor quantidade de gordura. Apresentou também diminuição da circunferência de cintura $(7 \mathrm{~cm})$, massa gorda e valores bioquímicos. Durante o estudo observou-se que, independentemente da composição da dieta, os valores reduzidos foram estatisticamente iguais para ambas ${ }^{15}$. Outro ponto importante avaliado foi a massa magra, que se manteve após o método de JI $(45,5 \pm 1,2$ para $46.1 \pm 1,6$ no grupo com ingestão maior de gordura e $46.1 \pm 1,6$ para $46.6 \pm 1.6$ no grupo com mais baixa ingestão de lipídeos), resultado semelhante ao descrito em outro estudo, que também registraram manutenção de massa magra nos participantes e redução de peso corporal $(5,2 \pm 0,9 \mathrm{~kg})^{16}$. 
De acordo com Hoddy et al. ${ }^{10}$, em seu estudo com 59 indivíduos de gêneros variados, utilizando uma restrição calórica (RC) de $25 \%$ em dias que a ingestão de alimentos é parcialmente reduzida, "dias rápidos", observou-se que, após 8 semanas de intervenção pelo JDA, houve uma redução no peso corporal significativa de aproximadamente $4 \mathrm{~kg}(93,5 \pm 1,4 \mathrm{~kg}$ a $89,7 \pm 1,5 \mathrm{~kg})$, no entanto, os demais parâmetros de circunferência de cintura, IMC e valores lipídicos não foram avaliados nesse estudo.

Clayton et al. ${ }^{17}$, realizaram uma pesquisa com dois ensaios tridimensionais com dietas padronizadas, sendo balanço energético (BE) de 100\% e restrição energética (RE) de $25 \%$, durante 3 dias. Eles perceberam um início de perda de peso de 1,1 kg entre o primeiro e segundo dia. Os pesquisadores também notaram uma alteração transitória no apetite e no aumento da ingestão em $7 \%$ nas primeiras 24 horas de $\mathrm{RE}$, mas após um ad libitum almoço no segundo dia, não houve diferença nas sensações subjetivas de apetite entre os ensaios. Além disso, 24 horas de severa restrição energética, não promoveram hormônios do apetite que são respostas indicativas de hiperfagia. Também ressaltaram que a ingestão média de energia no estudo foi $20 \%$ menor durante RE que durante BE. Além do mais, o estudo ainda sugeriu que períodos curtos de severa restrição energética podem ajudar a controlar $\mathrm{o}$ apetite em mulheres e homens magros.

Para jejum modificado foram identificados dois artigos em humanos. Em uma pesquisa de Harvie et al. ${ }^{18}$ realizada com mulheres saudáveis, com excesso de peso ou obesidade (faixa de IMC entre $24-45 \mathrm{Kg} / \mathrm{m}^{2}$ ) avaliou-se a composição corporal das participantes dividindo-as em três grupos de comparação, sendo: jejum modificado com restrição de carboidrato (JM+RCARB), jejum modificado com aumento na ingestão de proteínas e gorduras (JM+PG) e restrição de energia diária (RED). Pode-se perceber ao final de 4 meses, que houve uma perda significativa de peso de $5 \%$ entre os grupos de jejum intermitente comparando-se ao grupo de restrição energética diária. Além da perda de peso, foi observada a redução da circunferência de cintura em todos os grupos, porém os resultados do JM+RCARB e JM+PG foram mais significativos que o grupo RED. Com relação ao lipidograma, todos os grupos apresentaram reduções de colesterol total, LDL e triglicerídeos, com a manutenção do HDL. O mesmo estudo não apresentou a avaliação do IMC, mas observaram diferenças na composição de massa gorda (JM+RCARB= 31,0 para 26,7; $\mathrm{JM}+\mathrm{PG}=33,5$ para 29,4 e $\mathrm{RED}=35.7$ para 33,2 ). Como houve mudanças no peso no 
Marília Celeste Brito da Cunha, Edson Douglas Silva Pontes, Mayara Gabrielly Germano de Araújo, Jaielison Yandro Pereira da Silva, Nilcimelly Rodrigues Donato | A influência do método estratégico jejum intermitente para o emagrecimento saudável: uma revisão

decorrer de 4 meses, supõe-se que o IMC, consequentemente, também tenha reduzido.

Estudos apontam uma perda de peso de $5.3 \pm 3.0 \mathrm{~kg}$, após 6 meses de intervenção pelo método JM com 23 veteranos de guerra do sexo masculino e obesos, mesmo que durante os 5 dias restantes de alimentação/semana não aconteceu qualquer tipo de restrição dietética. Consequentemente, o IMC foi alterado de $33.4 \pm 1.8$ na linha de base para $31.5 \pm 2.2$ após 6 meses de intervenção ${ }^{11}$. Mudanças significativas na circunferência de cintura também foram relatadas, referentes à 8 a $10 \mathrm{~cm}$. Com relação ao lipidograma, o estudo apresentou valores mantidos, diferentemente de Harvie et al. $^{18}$, que embora em período mais curto de intervenção (4 meses), apresentou mudanças significativas.

Em nível religioso, foi realizado uma pesquisa de JIR durante o mês do Ramadã, com jejum de 14 horas consecutivas por dia em um período de 20 dias. $O$ trabalho avaliou a composição corporal comparando os resultados de acordo com o sexo da população e idade, porém, não foi considerada uma mudança significativa. Um outro parâmetro observado foi a perda de massa magra, que pode ser atribuído a uma taxa aumentada de degradação de proteína em relação à síntese proteica ${ }^{19}$.

De acordo com Harder-Lauridsen et al. ${ }^{20}$ após 8 semanas de intervenção ( 4 semanas de dieta controle mais 4 semanas de dieta por JI estilo Ramadã) com indivíduos saudáveis, onde a alimentação foi totalmente restrita por 14 horas ao dia, houve pequenas mudanças relacionadas ao peso corporal $(1.1 \mathrm{~kg})$, com diminuição da massa gorda total de $0,1 \%$ após 28 dias. 0 estudo relatou que a massa gorda perdida estava concentrada na região visceral. Observou-se também que houve diminuição de $1.0 \mathrm{~kg}$ de massa magra, porém, sem diferença estatisticamente significativa, mas que pode ser considerado como um efeito negativo. Com relação ao lipidograma, não houve nenhuma mudança nos valores e permaneceram constante após a pesquisa.

A JRT foi realizada em dois grupos, sendo um com 3 refeições diárias (grupo controle) e outro com 1 refeição diária por 4 horas a partir do início da noite (grupo experimental), durante 8 semanas. Ambos os grupos consumiram em torno de 1700 a $2100 \mathrm{kcal}$. Ao final do trabalho, constatou-se uma perda ponderal significativa de 1,4 kg após o Jejum por restrição de tempo, e, deste mesmo modo, redução da massa gorda de 2,1 kg. Observou-se também, que a massa magra se manteve durante o estudo e não sofreu nenhuma mudança significativa. Com relação ao lipidograma observou que a intervenção com 1 refeição por dia resultou em aumento do colesterol 
total e do LDL, porém, houve um aumento significativo do HDL e diminuição dos triglicerídeos ${ }^{21}$.

Lecheminant et al. ${ }^{22}$, apresentou um tempo diário de 14 horas consecutivas de abstinência total de qualquer alimento ou bebida com valor energético, em período noturno (19:0oh às 6:00h) e avaliou os efeitos de uma dieta de frequência reduzida para melhorias de saúde de uma população masculina jovem e saudável. Durante o turno diurno, fora de jejum, os participantes tiveram uma alimentação Ad libitum sem qualquer tipo de orientação. Após 2 semanas de protocolo pode-se constatar uma diminuição dos valores de peso corporal significativos (1.1 kg), assim como uma redução do IMC equivalente à $0.3 \mathrm{~kg} / \mathrm{m}^{2}$. Demais parâmetros de circunferência de cintura, lipidograma, massa gorda e massa magra não foram relatados no estudo.

\section{Viabilidade de execução das Intervenções}

Com relação a viabilidade, todas as subclasses de JI mostraram que não houve grande dificuldade dos participantes aderirem a dieta apontando que mais da metade da população inicial de todos os estudos conseguiram completar as intervenções. Alguns motivos de desistência, foram: Incapacidade de cumprir a dieta, conflitos de agendamento, razões pessoais e não especificadas ${ }^{10}$, desistência antes da intervenção $0^{14}$, incapacidade de cumprir protocolo e conflitos de agendamento ${ }^{15}$, intolerância a dieta e falta de disposição ${ }^{18,19}$, por motivos de viagens ${ }^{18,22}$ não gostaram da alimentação ofertada, conflitos de programação ${ }^{21}$ e motivos relacionados a saúde que não envolveram o estudo ${ }^{11,21}$.

Figura 1 - Nível de participação e desistência do JDA

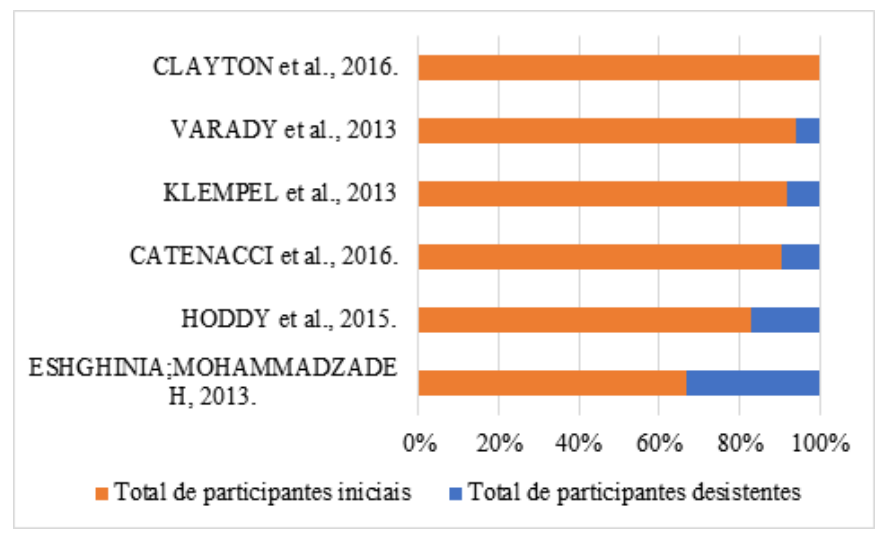


Marília Celeste Brito da Cunha, Edson Douglas Silva Pontes, Mayara Gabrielly Germano de Araújo, Jaielison Yandro Pereira da Silva, Nilcimelly Rodrigues Donato | A influência do método estratégico jejum intermitente para o emagrecimento saudável: uma revisão

Figura 2 - Nível de participação e desistência do JM

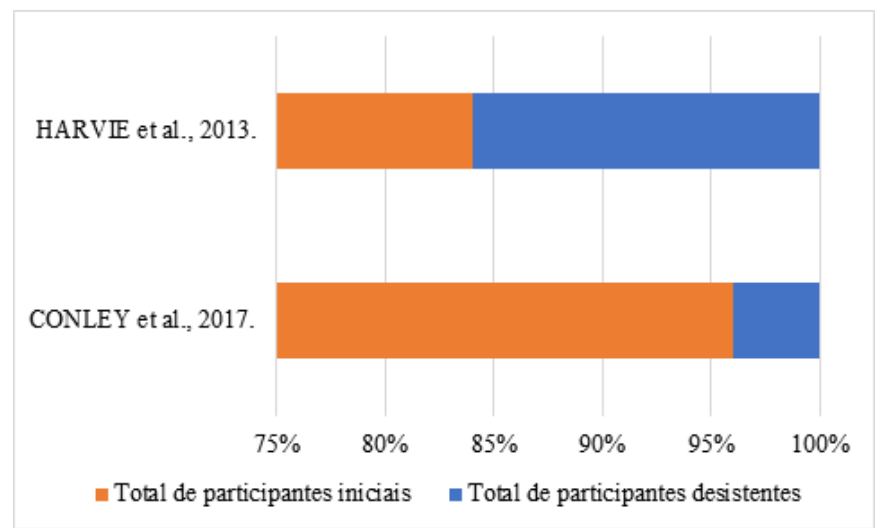

Figura 3 - Nível de participação e desistência do JIR

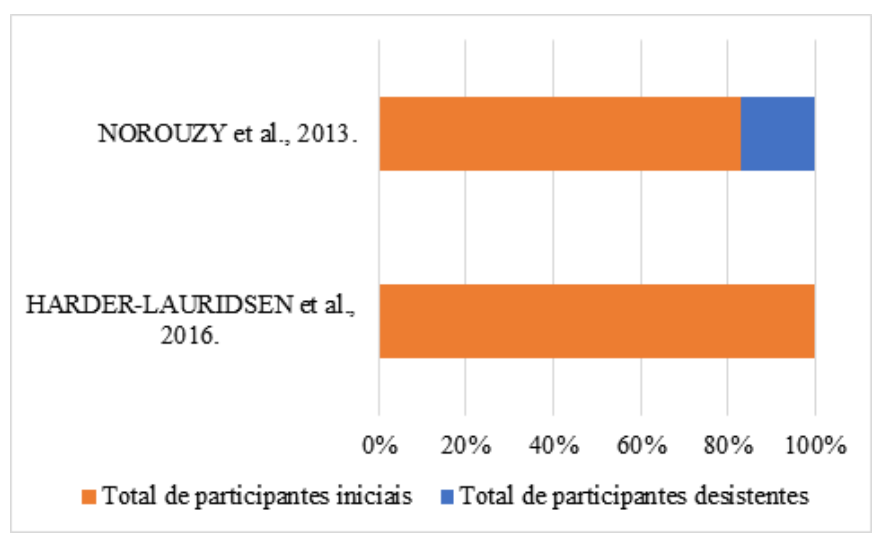

Figura 4 - Nível de participação e desistência do JRT

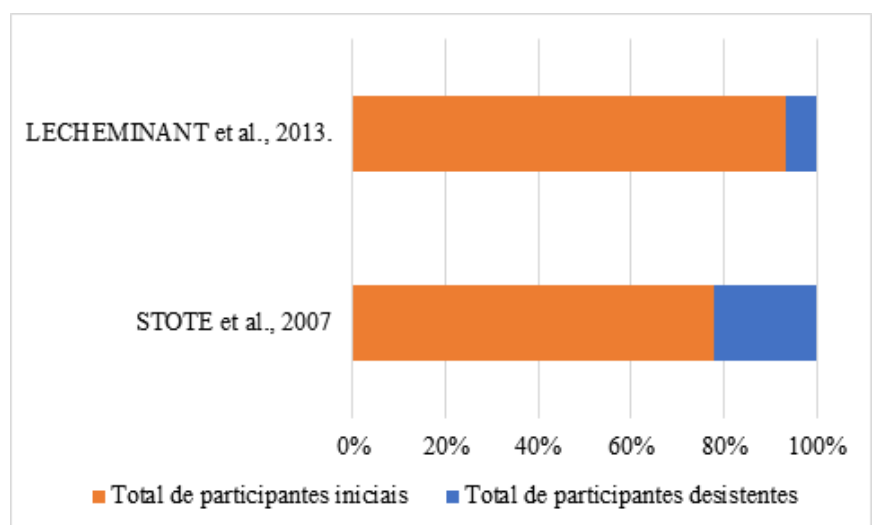

\section{Calorias totais consumidas}

No presente trabalho, todos os estudos sobre Jejum modificado e alguns de dia alternado, apresentaram intervenções que forneceram uma média de 25 a 30\% das necessidades diárias energéticas. Apenas 1 trabalho sobre JDA apresentou restrição calórica completa em "dia rápido", mas embora bons resultados sobre peso corporal 
Marília Celeste Brito da Cunha, Edson Douglas Silva Pontes, Mayara Gabrielly Germano de Araújo, Jaielison Yandro Pereira da Silva, Nilcimelly Rodrigues Donato | A influência do método estratégico jejum intermitente para o emagrecimento saudável: uma revisão

e aderência à dieta, não é sugestivo devido à baixa ingestão energética e inexistência de novos estudos para comparação ${ }^{23}$.

Quanto ao método JRT, todos os trabalhos apresentaram um valor calórico diário livre $^{21,22}$. Com relação ao JIR, os indivíduos de todos os estudos analisados abstiveram-se totalmente de comida ou bebida por 14 horas/dia durante o período do mês Ramadã ${ }^{19,20}$.

\section{Efeitos adversos}

Figura 5 - Frequência de relatos, de acordo com o número de estudos, dos efeitos adversos

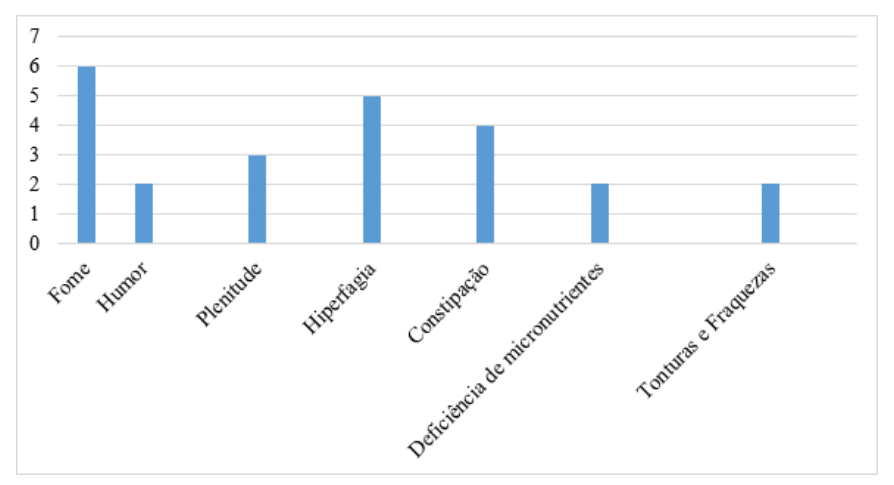

\section{Fome}

Considerando os 6 estudos que apresentaram uma avaliação do comportamento de fome, pode-se constatar que todos os participantes sentiram um acentuamento desse efeito, inicialmente, durante e após a intervenção JI. Em um estudo de jejum de dia alternado de 12 semanas, os níveis de fome foram moderados na linha de base da pesquisa e não apresentou mudanças significativas após a aplicação do método embora tenha havido uma redução leve $\left(5 \pm 1 \text { na } 1^{0} \text { semana e } 4 \pm 1 \text { na } 12^{\circ} \text { semana }\right)^{16}$. Em outro estudo de JDA, com período intervencional de 3 dias, a fome também aumentou, mais precisamente no segundo dia, embora que ao avaliar a quantidade do "hormônio da fome" (grelina) percebeu-se que os níveis estavam diminuídos ${ }^{17}$.

De acordo com um estudo de Jejum 5:2 de 4 meses, os resultados indicaram um aumento de fome durante o primeiro dia de protocolo de jejum modificado com restrição de carboidratos comparado ao protocolo de jejum modificado com aumento no consumo de proteínas e gorduras, sendo que após 3 e 4 meses as mulheres participantes do estudo se adaptaram com a intervenção11. Uma análise a ser feita desse estudo é que o tipo de macronutrientes fornecidos, assim como a qualidade da alimentação ofertada pode ajudar a reduzir a sensação de fome durante os primeiros dias de intervenção até obter uma resposta adaptativa. 
Estudos de JR também identificaram um aumento pronunciado de fome durante períodos de 28 dias, o que pode ser ocasionado pela falta de habituação com o método, tendo em vista que corresponde a uma intervenção de curto prazo, e a abstinência alimentar é total, tanto de líquidos como de alimentos ${ }^{20}$.

Assim como todas as outras subclasses de jejum, resultados de jejuns temporalizados apresentaram um aumento de fome. Os participantes de um determinado estudo, sentiram sensações de fome moderadamente maiores após um período de jejum restrito noturno de 19:00 horas às 06:00 horas, por 3 semanas, principalmente antes do café da manhã comparando-se com o grupo controle (sem restrição noturna). No entanto, não houve diferenças significativas entre as duas condições $^{22}$. Um outro estudo de 8 semanas com jejum por restrição de tempo, mostrou que a fome também foi identificada, principalmente no grupo que realizou esse tipo de JI consumindo apenas uma refeição diária comparado ao grupo que consumiu 3 refeições diárias ${ }^{21}$.

\section{Humor}

Dois artigos apresentaram a avaliação de alteração de humor durante intervenções de JI, correspondendo à um estudo de JR do Ramadã e outro sobre JRT. Com relação ao estudo de JR, os participantes apresentaram menor estado mental positivo durante a tarde do jejum20. Enquanto que, em uma intervenção de JRT esse efeito foi avaliado e não apresentou quaisquer alterações na linha de base, bem como, depois ${ }^{22}$.

\section{Plenitude}

A plenitude foi um efeito adverso relatado em três estudos sobre jejum de dia alternado e jejum por restrição de tempo. Esse efeito aumentou da linha de base para 12 semanas de intervenção de JDA, sendo uma mudança respectiva de $2 \pm 1$ para $4 \pm 116$. Entretanto, no estudo de apenas 3 dias, mostrou que a plenitude diminuiu no primeiro dia de intervenção em comparação ao grupo controle, no entanto, aumentou no decorrer dos dias ${ }^{17}$.

\section{Hiperfagia}

Um estudo de JDA durante o dia de alimentação rápida, apresentou pouca ou nenhuma resposta hiperfágica ${ }^{16}$. Outros dois estudos de JDA, mostraram uma diminuição desse efeito, diminuindo da linha de base para 8 semanas após a intervenção $(33 \pm 1 \text { para } 30 \pm 1)^{10}$ e em períodos muito curtos de três dias ${ }^{17}$. A respeito 
Marília Celeste Brito da Cunha, Edson Douglas Silva Pontes, Mayara Gabrielly Germano de Araújo, Jaielison Yandro Pereira da Silva, Nilcimelly Rodrigues Donato | A influência do método estratégico jejum intermitente para o emagrecimento saudável: uma revisão

de jejum por restrição de tempo, um estudo também identificou uma diminuição de hiperfagia através da redução da ingestão energética total ${ }^{22}$.

\section{Constipação}

A constipação foi um efeito adverso estudado por quatro pesquisas. Sobre Jejum de dia alternado, realizado com 30 participantes, apenas 1 participante, apresentou esse efeito durante a $1^{\mathrm{o}}$ e $2^{\mathrm{o}}$ semana de tratamento. Entretanto, após indicar o consumo de vegetais e frutas, houve uma diminuição dessa ocorrência9. Diferentemente do estudo de Hoddy et al. $^{10}$ o qual destacou que $17 \%$ dos participantes apresentaram esse efeito, mas nenhuma mudança ao decorrer do estudo foi notada. Em um outro trabalho, agora sobre jejum modificado, a constipação apareceu como uma suposição em resposta à baixa ingestão de fibras avaliada ${ }^{11}$. Bem como o estudo de Harvie et al. $^{18}$, no qual $8 \%$ dos participantes relataram apresentar constipação devido a diminuição da ingestão de fibras.

\section{Deficiência de micronutrientes}

Dois trabalhos sobre jejum com dieta 5:2 avaliaram a ingestão diária de micronutrientes. Um dos estudos relatou que houve uma deficiência de ingestão de cálcio, fato que faz pensar ser um método inviável, principalmente para população mais velha que precisa em maiores quantidades desse mineral11. O outro estudo registrou que houve deficiência de magnésio, ferro, zinco, cálcio, vitamina $A$ e $D$, que pode ter ocorrido pela estratégia do estudo ${ }^{18}$.

\section{Tonturas e fraquezas}

Poucos estudos avaliaram efeitos de tonturas e fraquezas. No presente trabalho foram identificadas duas pesquisas. A primeira pesquisa foi realizada a partir do protocolo da subclasse jejum de dia alternado, relatando que $<15 \%$ dos participantes apresentaram esses efeitos adversos sem qualquer alteração ao decorrer de 8 semanas10. Entretanto, em outro estudo sobre jejum por restrição de tempo foi relatado que esses efeitos tenderam a aumentar, mas estatisticamente a diferença não apresentou significância ${ }^{22}$.

\section{DISCUSSÃO}

Por meio dos achados científicos observou-se que o método jejum intermitente pode ser uma ferramenta viável, no entanto, muitos parâmetros devem ser minuciosamente estudados, dentre eles a massa gorda e massa magra, em vista que 
Marília Celeste Brito da Cunha, Edson Douglas Silva Pontes, Mayara Gabrielly Germano de Araújo, Jaielison Yandro Pereira da Silva, Nilcimelly Rodrigues Donato | A influência do método estratégico jejum intermitente para o emagrecimento saudável: uma revisão

alguns estudos relataram perda de peso significativas, mas não avaliaram a composição corporal ${ }^{11,17,22}$. Observou-se também que em um estudo de JDA, onde os pesquisadores avaliaram a composição da dieta ofertada aos participantes, sendo elas, com maior quantidade de lipídios (45\%) ou menor quantidade em relação ao outro grupo (25\%), porém com valor calórico total semelhante, percebeu-se que essa estratégia pode facilitar a perda de peso, a curto prazo, independente da composição gordurosa da dieta. Esses resultados têm várias implicações clínicas, por exemplo, indivíduos obesos precisarão ajustar de forma gradativa os tipos dos alimentos que ingerem, no início da dieta, modificando, assim, o padrão de consumo alimentar para experimentar a benefícios do JDA. Isso pode aumentar a adesão à dieta. Uma vez que o indivíduo tenha se ajustado ao padrão alimentar do JDA, recomenda-se modificar gradualmente para uma dieta com menor quantidade de gordura ( $<35 \% \mathrm{kcal}$ de gordura, $<7 \% \mathrm{kcal}$ de gordura saturada e $<200 \mathrm{mg} / \mathrm{d}$ de colesterol na dieta). Essa eventual mudança para uma dieta com conteúdo menor de gordura é importante, pois o consumo de uma dieta gordurosa/colesterol alto por um período maior foi associado ao aumento do risco de doença coronariana ${ }^{15}$.

A maioria dos programas de controle de peso usa restrição diária de energia, mas a restrição intermitente de energia foi sugerida como uma possível abordagem alternativa. Um estudo sobre JI modificado 5:2 avaliou sua funcionalidade para perda de peso e diminuição de circunferência de cintura em relação à restrição energética diária, e pode concluir que o JM é uma abordagem eficiente tanto quanto a restrição energética $^{18}$. No entanto, foram detectadas algumas divergências com relação ao lipidograma, as quais podem estar relacionadas com o sexo da população, idade e o estado físico dos participantes ${ }^{11,18}$.

Perdas de massa magra foram observadas durante protocolos de jejum intermitente religioso do Ramadã, no entanto, segundo os autores, provavelmente foi uma consequência de desidratação devido à abstinência de fluido ${ }^{20}$ ou pode ser atribuído a uma taxa aumentada de catabolismo proteico em relação à síntese proteica ${ }^{19}$. Embora as pequenas mudanças, estatisticamente não foram significativas, mostrando que os participantes conseguiram concluir a baixa frequência de refeições mantendo a energia total do corpo por um curto período de tempo. Isso também pode ser analisado como um efeito adaptativo do corpo humano no qual utiliza outras vias de obtenção de energia mantendo a homeostase ${ }^{20}$. 
Sobre JI temporizado/por restrição de tempo, foram encontrados poucos estudos na literatura recente com pesquisas realizadas em humanos que avaliaram parâmetros de composição corporal. Com bases nos dados relatados, percebe-se que um jejum com janela de restrição de 20 horas pode ser viável para perda de peso e mudanças na composição corporal. No entanto, o aumento em níveis de colesterol e LDL em comparação ao grupo controle sugere a importância de novos estudos para comparação já que, em contrapartida, o HDL aumentou e os triglicerídeos diminuíram. Entretanto, esse aumento do colesterol e LDL pode sugerir, também, uma resposta fisiológica do mecanismo da lipólise, já que, possivelmente, os triglicerídeos diminuíram por terem sido degradados para obtenção de ácidos graxos circulantes, aumentando consequentemente os valores lipídicos ${ }^{21}$. Portanto, faz-se necessário novos estudos que avaliem esses mecanismos nessas condições de jejum $^{23}$.

Tratando-se das calorias consumidas, algumas classificações como dieta de JM e dieta JDA possuem critérios que indagam alguns pontos, no que se refere a quantidade mínima de calorias consumidas durante os dias de intervenção, tendo em vista que restringem o valor calórico diário parcialmente (20 a 25\%; até $75 \%$, respectivamente) ou completamente (o calorias). A demanda energética diária para um homem sedentário é de aproximadamente 2.000 calorias e a quantidade de energia utilizada para realizar as atividades físicas diárias, normalmente é de cerca de $25 \%$ do gasto energético total podendo variar de acordo com a individualidade, do tipo de atividade física praticada e a quantidade ${ }^{23}$.

Sobre o JRT e calorias é importante ressaltar que o principal objetivo é restringir o tempo de ingestão de alimentos e não as calorias, logo a ingestão calórica é livre ${ }^{22,21}$. Já o JIR, refere-se a uma prática religiosa e não experimental o que só foi considerado no presente trabalho como uma fonte de curiosidade, dada a importância de estudar os efeitos do jejum intermitente em cada situação específica ${ }^{19,20}$.

Alguns estudos apontaram que o método JI em geral pode causar alguns efeitos adversos no organismo e os avaliaram em seus estudos 9 -11,16-18,20-22,24.

A fome é um efeito adverso que se apresentou em todas as intervenções, mas mostrou-se reduzida após alguns dias ou semanas de intervenção16 como uma resposta adaptativa ou pelo tipo de macronutriente ofertado ${ }^{11}$.

Poucos estudos avaliaram o efeito do humor após alguma intervenção de jejum intermitente, no entanto, os estudos encontrados mostraram que em curto prazo, o 
Marília Celeste Brito da Cunha, Edson Douglas Silva Pontes, Mayara Gabrielly Germano de Araújo, Jaielison Yandro Pereira da Silva, Nilcimelly Rodrigues Donato | A influência do método estratégico jejum intermitente para o emagrecimento saudável: uma revisão

humor pode sofrer algumas variações ${ }^{20,22}$. O humor foi avaliado em dois dias de medições após um protocolo de jejum intermitente e os resultados apresentaram um maior sentimento de humor negativo associado com maior distração, embora o sentimento de humor positivo foi maior na segunda medição em relação a primeira, sugerindo alguma tolerância ao jejum intermitente ${ }^{24}$.

Com relação à plenitude, foi descrito que ela também pode sofrer uma resposta adaptativa já que em ambos os trabalhos, na linha de base os valores eram reduzidos e aumentaram após as intervenções ${ }^{17}$.

Outra reação adversa é a hiperfagia, um efeito de fome excessiva que pode levar a compulsão alimentar. Muitos estudos desacreditam do método JI para perda de peso, pois ao restringir a alimentação por determinada densidade calórica ou tempo, pode induzir consequentemente o aumento pela busca do alimento e com isso obter um efeito negativo na composição corporal e saúde humana9 .

O presente trabalho mostrou que durante o JI houve diminuição da resposta hiperfágica em alguns estudos e ao apresentar esses dados nota-se que ao invés de provocar uma resposta negativa, o JI mostrou-se ser eficaz para diminuir a ingestão energética ${ }^{10,17}$. Dentre os trabalhos que avaliaram o efeito de hiperfagia, um trabalho sobre jejum temporizado, identificou um aumento hiperfágico durante a intervenção de1 refeição diária, fato que faz pensar na importância de novos estudos humanos com o mesmo método de intervenção para posteriormente comparar esse efeito ${ }^{22}$.

Outra consequência avaliada com o método JI foi a constipação. Os estudos apontaram que é possível que ao realizar o JI, os indivíduos podem apresentar esse efeito pela diminuição da ingestão de fibras, caso a conduta não seja realizada de forma correta pelo paciente ou se as recomendações diárias de fibras não sejam indicadas e reforçadas pelos profissionais ${ }^{9,10,11,18}$.

Assim como o humor, poucos estudos avaliaram as possíveis deficiências de micronutrientes, tonturas e fraquezas durante protocolos de JI. Pode-se observar que em relação à deficiência de micronutrientes, a estratégia utilizada tem influências nesses resultados e que se recomendações nutricionais forem realizadas adequadamente, minimizará consequentemente esses efeitos ${ }^{11,18}$. Tratando-se de tonturas e fraquezas, um estudo notou que poucos participantes sentiram esses efeitos10, entretanto, um estudo por JRT relatou um aumento desses efeitos, embora que estatisticamente a diferença não foi significativa, o que faz necessário novos estudos em ampla escala, diante de todas as subclasses de JI ${ }^{22}$. 
Apesar da existência de efeitos adversos em alguns estudos, a adesão dos participantes diante de todos os protocolos de JI foi boa, pois mais da metade da população inicial de todos os estudos conseguiram completar as intervenções.

\section{CONCLUSÃO}

Considerando os resultados obtidos, o método de JI pode ser uma estratégia promissora para a perda de peso em curto prazo quando utilizada de forma responsável por meio de acompanhamento nutricional adequado. As formas de JI, como a dieta de dia alternado parcial, jejum modificado e jejum por restrição de tempo mostraram ser fáceis de serem aplicadas, como também apresentaram efeitos positivos de mudanças corporais, em períodos de curto prazo. Entretanto, ressalta-se a importância de novas pesquisas envolvendo seres humanos para evidenciar de forma clara os reais benefícios do JI, seus efeitos nos valores bioquímicos de LDL, colesterol total e triglicerídeos, na massa gorda e magra e os efeitos adversos em cada subclasse desse método, sobretudo a longo prazo.

\section{REFERÊNCIAS}

1. Mattson MP, et al. Meal frequecy and timing in health and diase. Proceedings of The National Academy of Sciency of the United States of America. 2014;111(47):16647-16653.

2. Villain N, et al. Body mass loss correlates with cognitive performance in primates under acute caloric restriction conditions. Behavioural Brain Research. 2016;305: 157-163.

3. Horne BD, et al. Health effects of intermittent fasting: hormesis or harm? A systematic review. The American Journal of Clinical Nutrition. 2015;102(2):464470.

4. Persynaki A, et al. Unraveling the metabolic health benefits of fasting related to religious beliefs: A narrative review. Nutrition. 2017;35:14-20.

5. Eshghinia S, Mohammadzadh F, et al. The effects of modified alternate-day fasting diet on weight loss and CAD risk factors in overweight and obese women. Journal of Diabetes \& Metabolic Disorders. 2013;12(1):4.

6. Azevedo FR, et al. Effects of intermittent fasting on metabolism in men. Journal of the Brazilian Medical Association. 2013;59(2):167-173.

7. Longo VD, Mattson MP. Fasting: molecular mechanisms and clinical applications. Cell Metabolism. 2014;19 (2):181-192.

8. Mattson MP, et al. Meal frequency and timing in health and disease. Proceedings of the National Academy of Sciences. 2014;111(47):16647-16653.

9. Varady KA. Intermittent versus daily calorie restriction: which diet regimen is more effective for weight loss? Obesity reviews. 2011;12(7):593-601. 
10. Hoddy KK, et al. Safety of alternate day fasting and effect on disordered eating behaviors. Nutrition Journal. 2015; 14(1):44.

11. Conley $\mathrm{M}$, et al. Is two days of intermittent energy restriction per week a feasible weight loss approach in obese males? A randomised pilot study. Nutrition \& Dietetics: The Journal of the Dietitians Association of Austrália. 2017; 110:15341547.

12. Keogh JB, et al. Effects of intermittent compared to continuous energy restriction on short-term weight loss and long-term weight loss maintenance. Clinical Obesity. 2014; 4(3):150-156.

13. Sampaio RF, Mancini MC. Estudos de revisão sistemática: um guia para síntese criteriosa da evidência científica. Revista brasileira de fisioterapia. 2007;11(1):83-89.

14. Catenacci VA., et al. A randomized pilot study comparing zero-calorie alternateday fasting to daily caloric restriction in adults with obesity. Obesity, 2016;24(9):1874-1883.

15. Klempel MC. et al. Benefit of a low-fat over high-fat diet on vascular health during alternate day fasting. Nutrition \& Diabetes. 2017;3(5):1-6.

16. Varady KA, et al. Alternate day fasting for weight loss in normal weight and overweight subjects: a randomized controlled trial. Nutrition jornal. 2013;12(1):146.

17. Clayton DJ, et al. Effect of 24-h severe energy restriction on appetite regulation and ad libitum energy intake in lean men and women. The American Journal of clinical Nutrition. 2016;104(6):1545-1553.

18. Harvie M, et al. The effect of intermittent energy and carbohydrate restriction $\mathrm{V}$. daily energy restriction on weight loss and metabolic disease risk markers in overweight women. British Journal of Nutrition. 2013;8(110):1534-1547.

19. Norouzy A, et al. Effect of fasting in Ramadan on body composition and nutritional intake: a prospective study. Journal of Human Nutrition and Dietetics. 2013;26(1):97-104.

20. Harder-Lauridsen NM, et al. Ramadan model of intermittent fasting for $28 \mathrm{~d}$ had no major effect on body composition, glucose metabolism, or cognitive functions in healthy lean men. Nutrition.2017;37:92-103.

21. Stote KS, et al. A controlled trial of reduced meal frequency without caloric restriction in healthy, normal-weight, middle-aged adults. The American journal of clinical nutrition. 2007;85(4):981-98.

22. Lecheminant JD, et al. Restricting night-time eating reduces daily energy intake in healthy young men: a short-term cross-over study. British journal of nutrition. 2013;110(11):2108-2113.

23. Hall JE. Tratado de Fisiologia Médica. 12a ed. Rio de Janeiro Elsevier; 2011.

24. Appleton KM, Baker S. Distraction, not hunger, is associated with lower mood and lower perceived work performance on fast compared to non-fast days during intermittent fasting. Journal of health psychology. 2015;20 (6):702-711.

Submissão: $16 / 05 / 2018$

Aprovação: 05/12/2020 